\title{
HORNER'S SYNDROME IN A CONGENITAL SCOLIOSIS PATIENT: A COMPLICATION RELATED TO INTERNAL JUGULER VEIN CATHETERISATION OR PRONE POSITION?
}

\author{
Başkent University Faculty of Medicine, Adana Dr. Turgut Noyan Application and Research Center, Department of Anesthesiology
} and Reanimation, Adana, Turkey

\begin{abstract}
Horner's syndrome (HS) is a rare complication after anterior cervical discectomy and it is characterized by ipsilateral ptosis, miosis and fascial anhidrosis. Here, we described transient postoperative HS in a patient with congenital scoliosis who underwent posterior T7 hemivertebra excision. Fortunately, HS resolved completely after 3 months of follow-up. One must consider internal jugular vein catheterization or prone position as the cause of postoperative HS in non-cervical surgeries.
\end{abstract}

Keywords: Horner's syndrome, jugular vein catheterization, prone position, congenital scoliosis

\section{INTRODUCTION}

Postoperative Horner's syndrome (HS) is a rare complication characterized by ipsilateral ptosis, miosis and facial anhidrosis. It is the result of direct or indirect compression or tension of oculosympathetic fibers ${ }^{(1)}$. Anterior cervical discectomy and fusion surgery is the most common cause of direct trauma ${ }^{(2)}$. Additionally, the syndrome can occur as a result of recurrent attempts of needle damage during internal jugular vein (IJV) catheterization. In our case, we reported postoperative HS in a congenital scoliosis patient who underwent posterior T7 hemivertebra excision with a history of IJV catheterization.

\section{CASE REPORT}

The 12-year-old-girl patient underwent posterior T7 hemivertebra excision and posterior instrumentation and fusion between T1 and T10 levels. After uneventful anesthesia induction, the correct head position was given, and landmark method is used for right IJV catheterization. We succeeded IJV catheterization after three unsuccessful attempts. After standard monitoring and arterial monitoring, the patient was turned to the prone position. Operation time was 240 minutes; the patient was extubated uneventfully.
At the postoperative $1^{\text {st }}$ day, we recognized that the right site pupil was smaller than the left one. On physical examination, we detected ptosis and miosis on the right site, and HS was diagnosed. Neurology consultation was done. Bilateral light reaction and cranial nerve examination were normal. No pathologic finding was detected in her cervical magnetic resonance imaging study. There was no cervical hematoma, and her range of motion was normal. No medication was given to the patient. The HS resolved completely after 3 months followup.

\section{DISCUSSION}

HS is the result of the interruption of an oculosympathetic pathway to the eye with different causes. Sympathetic preganglionic fibers exit from high centers like the hypothalamus and spinal cord and descend across the neck with cervical sympathetic chain. After they make synapse with superior cervical ganglion at the back site of carotis artery, postganglionic fibers enter the orbita next to the internal carotis artery.

IJV is located at anterolateral site of cervical sympathetic chain in normal anatomy. Because of the close relationship during IJV catheterization, injection damage or hematoma compression 
caused by unexpected puncture of carotis, it can effect cervical sympathetic branches and stellate ganglion ${ }^{(3)}$. Damage risk is high with recurrent unsuccessful attempts. Anatomic variations and with turning the head over $40^{\circ}$ makes IJV and carotis artery to overlap, so the risk of artery puncture and damage to sympathetic brunch increase ${ }^{(4)}$. At this point, ultrasonography usage increases the success of the process, and unnecessary arterial puncture and hematoma formation and damage of sympathetic brunches are prevented. In the literature, there are case reports in which complications reported despite ultrasonography usage ${ }^{(3)}$. Other cause of direct trauma is surgery. Cervical spinal surgery and carotis endarterectomy are high-risk ones but it can occur in other surgeries involving cervical area $a^{(2,5)}$.

Lubelski et al.(6) performed a retrospective systemic review including 1,116 patients who passed anterior cervical spinal surgery. Because of the anatomic location of sympathetic brunches, C4-5 and C5-6 levels were the most common surgical levels causing HS. They reported higher incidence than the literature reported ${ }^{(6)}$. Generally, local anesthetic diffusion after brachial plexus blockage or high levels in lumbar/thoracal epidural anesthesia are the examples of postoperative HS due to anesthesia applications. These are generally transient cases $^{(7)}$.

HS can occur in decubitus and supine positions where the neck position is not in neutral position ${ }^{(8)}$. Here the mechanism is excess stretching of sympathetic chain with exaggerated positions and resulting with secondary ischemia. HS in the prone position was also reported in the literature ${ }^{(9)}$. In one study, the authors presented a patient who underwent a 6-hour abdominoplasty and liposuction surgery and developed postoperative HS. Surgery had three phases, and the first phase lasted for two hours in the prone position. Authors related this complication to stretching and compression of the oculosympathetic pathway in the upper part of the thorax during the prone position. In a prone position, there is prolonged excessive pressure on the thorax and with abduction of the arms, greater pressure exerted on the thorax ${ }^{(9)}$. Prolonged operation time may be the other additional cause.

\section{CONCLUSION}

There is no specific treatment of HS so prevention is so important. Recurrent attempts with landmark method, excess rotation of the head to the left site, exaggerated positions, fluid-drug extravasations or hematoma due to carotis puncture are the risk factors for HS. In our case, cause of the HS can be direct puncture of the needle due to recurrent attempts or prone position in a long operation surgery.

One must consider IJV catheterization as the cause of postoperative HS in non-cervical surgeries.

\section{Ethics}

Informed Consent: Informed consent was obtained from the patient.

Peer-review: Internally peer-reviewed.

Financial Disclosure: The author declared that this study did not receive any financial support.

\section{REFERENCES}

1. Walton KA, Buono LM. Horner syndrome. Curr Opin Ophthalmol. 2003; 14:357-63.

2. Civelek E, Karasu A, Cansever T, Hepgul K, Kiris T, Sabanci A, et al. Surgical anatomy of the cervical sympathetic trunk during anterolateral approach to cervical spine. Eur Spine J. 2008;17:991-5.

3. Zou ZY, Yao YT. Horner syndrome caused by internal jugular vein catheterization. Cardiothorac Vasc Anesth. 2020;34:1636-40.

4. Sulek CA, Gravenstein N, Blackshear RH, Weiss L. Head rotation during internal jugular vein cannulation and the risk of carotid artery puncture. Anesth Analg. 1996;82:125-8.

5. Hidayat H, Medani M, Kavanagh E. Transient iatrogenic Horner's syndrome following carotid endarterectomy. BM] Case Rep 2019;12:e228244. doi: 10.1136/bcr-2018-228244.

6. Lubelski D, Pennington Z, Sciubba DM, Theodore N, Bydon A. Horner syndrome after anterior cervical discectomy and fusion: case series and systematic review. World Neurosurg. 2020;133:68-75.

7. Ramez B, Riad T, Ankur B, Adel B, Luis G, Marwan O, et al. Transient Horner syndrome following epidural anesthesia for labor: case report and review of the literature. Obstet Gynecol Surv. 2011;66:114-9.

8. Jaffe TB, McLeskey $\mathrm{CH}$. Position-induced Horner's syndrome. Anesthesiology. 1982;56:49-50.

9. Guillaume JE, Gowreesunker P. Horner's syndrome in the prone position- a case report. Acta Anaesthesiol Belg. 2013;64:119-21. 